\title{
Structure and dynamics of the Shapley Supercluster ${ }^{\star}$
}

\section{Velocity catalogue, general morphology and mass}

\author{
D. Proust ${ }^{1}$, H. Quintana ${ }^{2}$, E. R. Carrasco ${ }^{3}$, A. Reisenegger ${ }^{2}$, E. Slezak ${ }^{4}$, H. Muriel $^{5}$, \\ R. Dünner ${ }^{2}$, L. Sodré Jr. ${ }^{6}$, M. J. Drinkwater ${ }^{7}$, Q. A. Parker ${ }^{8}$, and C. J. Ragone
}

1 GEPI, Observatoire de Paris-Meudon, 92195 Meudon Cedex, France

e-mail: dominique.proust@obspm.fr

2 Departamento de Astronomía y Astrofisica, Pontificia Universidad Católica de Chile, Casilla 306, Santiago 22, Chile

3 Gemini Observatory, Southern Operations Center c/o AURA, Casilla 603, La Serena, Chile

4 Observatoire de Nice, 06304 Nice Cedex 4, France

5 Grupo de Investigaciones en Astronomía Teórica y Experimental, Observatorio Astrónomico, Laprida 854, 5000 Córdoba, Argentina, and CONICET, Buenos Aires, Argentina

6 Instituto de Astronomia, Geofísica e Ciências Atmosféricas, R. do Matão 1226, CEP 05508-090 São Paulo/SP Brazil

7 Department of Physics, University of Queensland, QLD 4072, Australia

8 Department of Physics, Macquarie University, NSW 2109, Australia, and Anglo-Australian Observatory, PO Box 296, Epping NSW 1710, Australia

Received 8 February 2005 / Accepted 28 September 2005

\section{ABSTRACT}

We present results of our wide-field redshift survey of galaxies in a 285 square degree region of the Shapley Supercluster (SSC), based on a set of 10529 velocity measurements (including 1201 new ones) on 8632 galaxies obtained from various telescopes and from the literature. Our data reveal that the main plane of the SSC $\left(v \approx 14500 \mathrm{~km} \mathrm{~s}^{-1}\right)$ extends further than previous estimates, filling the whole extent of our survey region of 12 degrees by 30 degrees on the sky $\left(30 \times 75 \mathrm{~h}^{-1} \mathrm{Mpc}\right)$. There is also a connecting structure associated with the slightly nearer Abell 3571 cluster complex $\left(v \approx 12000 \mathrm{~km} \mathrm{~s}^{-1}\right)$. These galaxies seem to link two previously identified sheets of galaxies and establish a connection with a third one at $\bar{v}=15000 \mathrm{~km} \mathrm{~s}^{-1}$ near $\mathrm{RA}=13^{\mathrm{h}}$. They also tend to fill the gap of galaxies between the foreground Hydra-Centaurus region and the more distant SSC. In the velocity range of the Shapley Supercluster $\left(9000 \mathrm{~km} \mathrm{~s}^{-1}<c z<18000 \mathrm{~km} \mathrm{~s}^{-1}\right.$ ), we found redshift-space overdensities with $b_{j}<17.5$ of $\simeq 5.4$ over the 225 square degree central region and $\simeq 3.8$ in a 192 square degree region excluding rich clusters. Over the large region of our survey, we find that the intercluster galaxies make up 48 per cent of the observed galaxies in the SSC region and, accounting for the different completeness, may contribute nearly twice as much mass as the cluster galaxies. In this paper, we discuss the completeness of the velocity catalogue, the morphology of the supercluster, the global overdensity, and some properties of the individual galaxy clusters in the Supercluster.

Key words. galaxies: clusters: individual: Shapley Supercluster - galaxies: clusters: individual: A 3558 - galaxies: distances and redshifts cosmology: observations - cosmology: large-scale structure of Universe - galaxies: clusters: general

\section{Introduction}

In the past few decades, large galaxy redshift surveys have revealed structures on ever-increasing scales. The largest coherent structures found are superclusters, collections of thousands of galaxies with linear sizes as large as $100 \mathrm{Mpc}$. They offer us information about the late evolution of the Universe and the transition from the linear to the non-linear regime. Detailed investigations have shown the reality of such physical systems

^ Based on observations made at the European Southern Observatory, La Silla, Chile, at the Las Campanas Observatory, Chile, and at the Anglo-Australian Observatory, Australia. as gravitationally assembling clusters. Galaxy distributions and weak lensing showed that the mass distribution in superclusters is in good agreement with the distribution of early-type galaxies in these structures (Einasto et al. 2003a; Oguri et al. 2004). The mere existence of these structures places important constraints on theories of the formation of galaxies and clusters. The Shapley Supercluster, the subject of this paper, is one of the most massive concentrations of galaxies in the local Universe (Scaramella et al. 1989; Raychaudhury 1989). It therefore deserves a special examination of its organisation and it is also of particular interest to consider its effect on the dynamics of the Local Group. 
The Shapley Supercluster (SSC) is a remarkably rich concentration of galaxies centred around RA $=13^{\mathrm{h}} 25^{\mathrm{m}}$, Dec $=-30^{\circ}$, which has been investigated by numerous authors since its discovery in 1930 (see Quintana et al. 1995, 2000). It consists of many clusters and groups of galaxies in the redshift range $0.04<z<0.055$. The SSC lies in the general direction of the dipole anisotropy of the Cosmic Microwave Background (CMB; Smoot et al. 1992), and is located $\sim 100 h^{-1}$ Mpc beyond the Hydra-Centaurus supercluster, which in turn is $\simeq 40 h^{-1} \mathrm{Mpc}$ away from us (with the Hubble constant parameterized as $H_{0}=100 h \mathrm{~km} \mathrm{~s}^{-1} \mathrm{Mpc}^{-1}$ ). Quintana et al. (1995) estimated that (for $\Omega_{\mathrm{M}}=0.3, \Omega_{\Lambda}=0.7$, and $H_{0}=75 \mathrm{~km} \mathrm{~s}^{-1} \mathrm{Mpc}^{-1}$ ) the gravitational pull of the supercluster may account for up to $25 \%$ of the peculiar velocity of the Local Group required to explain the CMB dipole anisotropy, in which case the mass of the supercluster would be dominated by intercluster dark matter. A major study of the SSC core region was made by Bardelli et al. (2000, 2001 and references therein). They derived a total mean overdensity of the SSC of $N / \bar{N} \sim 11.3$ on a scale of $10.1 h^{-1} \mathrm{Mpc}$ and found that the central part of the supercluster contributes about $26 \mathrm{~km} \mathrm{~s}^{-1}$ to the peculiar velocity of the Local Group, i.e., $7 \%$ of the total tidal bulk velocity of $366 \pm 125 \mathrm{~km} \mathrm{~s}^{-1}$ from Hoffman et al. (2001). The central cluster A3558 has also been observed with the ROSAT PSPC by Bardelli et al. (1996), who derive a total mass of $M_{\mathrm{tot}}=3.1 \times 10^{14} h^{-1} M_{\odot}$ within a radius of $2 h^{-1} \mathrm{Mpc}$. Several other luminous X-ray clusters are part of the Shapley Supercluster (Pierre et al. 1994).

The early studies of the Shapley Supercluster were limited (primarily by observational constraints) to the various rich Abell galaxy clusters in the region. This approach might give a very biased view of the overall supercluster, as these clusters represent only the most concentrated peaks in the luminous matter distribution. An analysis based on 3000 galaxy redshifts in an area of about $12 \mathrm{deg} \times 8 \mathrm{deg}$ (Quintana et al. 2000) was published by Reisenegger et al. (2000). They estimated an upper bound on the mass of the central region (within a radius of $8 h^{-1} \mathrm{Mpc}$ ) and found the overdensity to be substantial, but insufficient to contribute more than a small fraction $\left(3 \Omega_{m}^{-0.4} \%\right)$ of the observed motion of the Local Group.

In this paper, we make an investigation of the large-scale distribution of galaxies within the entire SSC region and nearby regions, using data from long-slit and wide-field multi-fiber spectrographs. Most of these observational data have already been published. A large survey was carried out over several years with S. Shectman's fiber spectrograph mounted on the 100" DuPont telescope at Las Campanas Observatory (LCO). Quintana et al. (2000) reported 2868 new velocities measurements, corresponding to 2627 different galaxies observed at LCO. The complete LCO data, which represent $40 \%$ of the catalogue, are being published (Quintana et al. 2005), including a new (and last) set of 1201 velocities on 1166 galaxies observed with the same instrument between 1997 and 1999. Another fraction of redshifts has come from the FLAIR facility of the UKST at the Anglo Australian Observatory (AAO). With such multiplexed facilities, we were able to measure more galaxy redshifts over large angular extents and obtain a more complete picture of the composition and distribution of galaxies in the entire supercluster. Combined with already published redshift sets from several surveys and papers (Quintana et al. 1995, 1997, 2000; Bardelli et al. 1996, 1998, 2001; Drinkwater et al. 1998, 1999, 2004; the FLASH survey of Kaldare et al. 2003, and the 6dF survey of Jones et al. 2004), we built up the most complete velocity catalogue for the SSC, containing 10529 velocity measurements for 8632 galaxies.

The complete galaxy velocity catalogue is described in Sect. 2. This section also includes comparisons between the galaxies in common among different data sets, as well as the velocity zero-point shifts required to end up with a homogeneous catalogue at our disposal. In Sect. 3, we discuss the completeness of this catalogue and analyse the galaxy number density over the whole and the intercluster survey regions. In Sect. 4 we analyse the three-dimensional morphology of the SSC. In Sect. 5, we discuss properties of the individual Abell clusters, and in Sect. 6 we determine the global luminosity and mass of the SSC and its contribution to the peculiar motion of the Local Group with respect to the cosmic microwave background.

\section{The velocity catalogue}

We investigated the large-scale distribution of galaxies throughout the entire SSC region as defined in Quintana et al. (2000) and close environs using data from long-slit and widefield multi-fibre spectrographs at various telescopes. Most of these data are related to 3 major surveys: $40 \%$ from Las Campanas observations (Quintana et al. 2005), 24\% from the UKST at Siding-Spring, Australia (Drinkwater et al. 1999; 2004; Kaldare et al. 2003; Jones et al. 2004), 15.5\% from the ESO 3.6 m telescope at La Silla, Chile (Bardelli et al. 1997, 1999, 2001; Quintana et al. 1997). We also searched the literature for velocities between $11^{\mathrm{h}} 30^{\mathrm{m}}<\mathrm{RA}<14^{\mathrm{h}} 30^{\mathrm{m}}$ and $-23^{\circ}>$ Dec $>-45^{\circ}$. A total of 1520 galaxies have been observed more than once, which allows us both to obtain more accurate velocity determinations for these galaxies and to compare different data sets for assessment of the general quality of the data and shifts in the velocity zero-point. Combined with already published redshift sets from several surveys and papers, we obtain the most complete velocity catalogue for the SSC area, containing 10529 velocities for 8632 galaxies, which are now available from the NED database ${ }^{1}$. The main point before any analysis is to combine these different data sets to build a homogeneous catalogue. Below, we comment on the detailed sources and instruments used in this catalogue.

\subsection{Las Campanas data}

The spectroscopic observations were carried out using the fiber spectrograph mounted on the $2.5 \mathrm{~m}\left(100^{\prime \prime}\right)$ du Pont telescope at Las Campanas Observatory ${ }^{2}$ in several observing sessions ranging from 1992 to 1999 . Some of them are already published in Quintana et al. (1995, 1997). 2868 new measurements

\footnotetext{
1 The NASA/IPAC Extragalactic Database (NED) is operated by the Jet Propulsion Laboratory, California Institute of Technology, under contract with the National Aeronautics and Space Administration.

2 The Las Campanas Observatory is operated by the Carnegie Institution of Washington.
} 
are described and compared with other sources in Quintana et al. (2000). A set of 1201 new velocities for 1166 different galaxies has been obtained between 1997 and 1999. All these data are described and published in Quintana et al. (2005).

\subsection{UKST/FLAIR-II, $6 d F$ and FLASH data}

Two sets of published data were obtained with the UKST/FLAIR-II system at the Anglo Australian Observatory (Drinkwater et al. 1999, 2004). They correspond to 710 galaxies observed over 7 UKST standard fields in the SSC region, covering an area of $182 \mathrm{deg}^{2}$. The target galaxies were originally obtained from the red ESO/SRC sky survey plates scanned by the Paris Observatory MAMA plate-measuring machine. The galaxy sample was defined to a photometric limit of $R=16$, corresponding (assuming a mean $B-R=1.5$ ) to $B=17.5 \approx b_{j}$, the nominal galaxy limiting magnitude of the FLAIR-II system (Parker \& Watson 1995). This corresponds to an absolute magnitude of $M_{B}=-18.3+5 \log h$ at the Shapley distance of $145 h^{-1} \mathrm{Mpc}$. The data were reduced using the dofibers package in IRAF (Tody 1993). Redshifts were measured for galaxy spectra using the cross-correlation task XCSAO in RVSAO (Kurtz \& Mink 1998) using a mixture of a dozen stellar and galaxy templates.

The Six-Degree Field Galaxy Survey (6dFGS) of the Southern sky, currently in progress, aims to measure the redshifts of around 150000 galaxies, and the peculiar velocities of a 15000 galaxy sub-sample, over almost the entire southern sky (Jones et al. 2004). Among this large target sample, 1328 remaining candidates from the above FLAIR-II program were selected in the SSC area. The 6dF fibre spectroscopy facility installed at the UKST has up to now observed 584 galaxies in the Shapley region with reliable redshifts. The reduction of the spectra was made using an adapted version of the $2 \mathrm{dF}$ Galaxy Redshift Survey software (Colless et al. 2001).

To complete the set of velocities obtained from the UKST, we have also included observations from the FLASH (FLAIR Shapley-Hydra) redshift survey of Kaldare et al. (2003). It consists of 4613 galaxies brighter than $b_{j}=16.7$ over a 700 square degree region of sky in the general direction of the Local Group motion. The survey region is a $70^{\circ} \times 10^{\circ}$ strip spanning the sky from the SSC to the Hydra cluster and contains 3141 galaxies with measured redshifts. The reduction procedure was similar to that used above for FLAIR-II spectra (Drinkwater et al. 1999, 2004).

After eliminating already published velocities from NED and galaxies outside the SSC region, we add 1411 new velocities to our database.

\subsection{ESO observations}

A major study of the SSC was made by Bardelli et al. (1994, 1996, 1998, 2000, 2001) and Baldi et al. (2001). All their observations were made at the ESO, La Silla (Chile) $3.6 \mathrm{~m}$ telescope, initially equipped with the multiobject facility OPTOPUS (Lund 1986), and later with MEFOS (Felenbok et al. 1997). Their analyses are based on data from the literature and on
Table 1. Comparison of the velocities and Zero Point shift between new velocities and data already published.

\begin{tabular}{lrrrrr}
\hline \hline References & $N_{\text {ref }}$ & $N_{\text {comm }}$ & $\begin{array}{r}\Delta v \\
\mathrm{~km} \mathrm{~s}^{-1}\end{array}$ & $\begin{array}{r}\sigma_{\Delta v} \\
\mathrm{~km} \mathrm{~s}^{-1}\end{array}$ & $\begin{array}{r}\mathrm{rms} \\
\mathrm{km} \mathrm{s}^{-1}\end{array}$ \\
\hline Bardelli et al. (2001) & 581 & 48 & -6 & 15 & 98 \\
Kaldare et al. (2003) & 1411 & 82 & -1 & 21 & 103 \\
Drinkwater et al. (2004) & 404 & 17 & 11 & 15 & 61 \\
Jones et al. (2004) & 584 & 29 & 8 & 13 & 73 \\
Quintana et al. (2005) & 1201 & 39 & 18 & 23 & 86 \\
\hline
\end{tabular}

4 sets of new velocities, 311 in Bardelli et al. (1994), 174 in Bardelli et al. (1998), 442 in Bardelli et al. (2000), then 662 for 581 new galaxies in Bardelli et al. (2001), with a total of 1589 new velocities, representing $15.5 \%$ of the velocity catalogue. Their sample is homogeneous, in a well defined magnitude range $\left(17.0<b_{j}<18.8\right)$. Their analyses are mainly concerning the SSC central region based on a sample of $\simeq 1300$ spectra covering an area of $\simeq 26 \mathrm{deg}^{2}$.

Another survey centered on 15 Abell cluster targets in the direction of the SSC has been carried out by our group (Quintana et al. 1997), also using the multiobject facility MEFOS at the ESO $3.6 \mathrm{~m}$ telescope. An additional set of 179 velocities has been obtained and is included in our general database.

\subsection{Comparison of the velocities}

Comparison of the velocities was made using the 1520 galaxies of the database with more than one velocity measurement. In order to combine the new data described above with those already available in the NED database, we normalized all radial velocity sets to a common zero point as in Quintana et al. (2000). If the zero points of different instruments differed by significant amounts, we could have introduced serious systematic errors in the resulting velocity dispersion and in the dynamical analysis. Table 1 summarizes the results of the zeropoint shifts obtained from comparison of the new data with the previously published ones, similar to Table 2 in Quintana et al. (2000). Finally, we have applied the zero-point shifts given in Table 1 to all velocities of the catalogue, before combining the measurements available for each galaxy into a final average value. For references without velocities in common with our data, or when the number of velocities was to low to obtain a meaningful shift, we compared the data with other datasets in the literature, and calculated the zero-point shift in a transitive way. The average velocity for galaxies with multiple references was calculated following Quintana et al. (2000). In a few cases, we discarded a velocity when analysis of the spectra showed discrepancies which differed by more than $200 \mathrm{~km} \mathrm{~s}^{-1}$. The final sample resulting from this procedure contains 8632 galaxies.

\section{Observed galaxy sample, completeness and overdensity}

For the purpose of our analysis below, we compare the velocity catalogue to the total magnitude-limited galaxy distribution 
Table 2. Completeness for each of the 9 Shapley ESO/UKST fields for four $b_{j}$ magnitude limits (all galaxies brighter than the given limit).

\begin{tabular}{|c|c|c|c|c|c|}
\hline Name & $\overline{\text { F446 }}$ & $\overline{\text { F445 }}$ & $\overline{\text { F444 }}$ & $\overline{\text { F443 }}$ & $\overline{\text { F442 }}$ \\
\hline RA & $14^{\mathrm{h}} 11^{\mathrm{m}}$ & $13^{\mathrm{h}} 48^{\mathrm{m}}$ & $13^{\mathrm{h}} 25^{\mathrm{m}}$ & $13^{\mathrm{h}} 02^{\mathrm{m}}$ & $12^{\mathrm{h}} 39^{\mathrm{m}}$ \\
\hline Dec & $-30^{\circ}$ & $-30^{\circ}$ & $-30^{\circ}$ & $-30^{\circ}$ & $-30^{\circ}$ \\
\hline$b_{j}<17.0$ & 0.29 & 0.67 & 0.92 & 0.94 & 0.33 \\
\hline$b_{j}<17.5$ & 0.17 & 0.48 & 0.79 & 0.79 & 0.20 \\
\hline$b_{j}<18.0$ & 0.10 & 0.30 & 0.61 & 0.62 & 0.10 \\
\hline$b_{j}<18.5$ & 0.06 & 0.19 & 0.44 & 0.43 & 0.07 \\
\hline Name & & F384 & F383 & F382 & F381 \\
\hline RA & & $4^{\mathrm{h}} 00^{\mathrm{m}}$ & $13^{\mathrm{h}} 36^{\mathrm{m}}$ & $13^{\mathrm{h}} 12^{\mathrm{m}}$ & $12^{\mathrm{h}} 48^{\mathrm{m}}$ \\
\hline Dec & & $-35^{\circ}$ & $-35^{\circ}$ & $-35^{\circ}$ & $-35^{\circ}$ \\
\hline$b_{j}<17.0$ & & 0.33 & 0.65 & 0.60 & 0.26 \\
\hline$b_{j}<17.5$ & & 0.19 & 0.51 & 0.48 & 0.16 \\
\hline$b_{j}<18.0$ & & 0.10 & 0.29 & 0.33 & 0.11 \\
\hline$b_{j}<18.5$ & & 0.07 & 0.18 & 0.21 & 0.05 \\
\hline
\end{tabular}

in the survey region. We chose to use the new SuperCOSMOS sky surveys (Hambly et al. 2001a,b,c) to construct the parent photometric galaxy catalogue for the whole region covered by our nine fields observed with UKST. We could cross-correlate almost each object with a SuperCOSMOS galaxy within a matching radius of 8 arcsec. The $6 \%$ remaining galaxies were visually checked on the sky plates. In this paper, we quote and use in the analyses the SuperCOSMOS $b_{j}$ magnitudes of all galaxies. We compared the magnitude distributions of the objects identified in the nine UKST fields, 381 to 384 and 442 to 446: two-sample K-S tests showed that they were all consistent with the same distribution (mean $b_{j} \simeq 17.2$ ).

Allowing for the boundaries of the survey fields and a circular region 1 degree in diameter, which we excluded around the bright star HD 123139 (at RA $=14^{\mathrm{h}} 06^{\mathrm{m}} 41^{\mathrm{s}}$, Dec $=$ $-36^{\circ} 22^{\prime} 12^{\prime \prime}$, J2000), the total survey region has an area of 225 square degrees. We also defined a restricted intercluster region by excluding regions 1 degree in diameter around any rich galaxy clusters in the Shapley velocity range, leading to an area of 192 square degrees.

\subsection{Completeness of the velocity catalogue}

In such a compilation, it is evident that different authors had different, often not easily quantifiable selection criteria. This fact makes the completeness analysis given here necessary, but, on the other hand, it makes it impossible to do it perfectly, because the many biases cannot be fully grasped by the variables RA, Dec, magnitude and cluster membership. Thus, some biases (such as the one due to morphology) are necessarily left even after completeness corrections are done.

We used the parent photometric galaxy catalogue to determine the completeness of the velocity catalogue as a function of 4 limiting magnitudes, respectively $b_{j}=17.0,17.5,18.0$, and 18.5. This is shown in Table 2 for each UKST plate and in Table 3 for both the full region and the intercluster region. This table shows that the completeness is highest for the brighter magnitude limits, peaking at $61 \%$ for the whole sample at
Table 3. Global completeness of the velocity catalogue for $4 b_{j}$ magnitude limits. The completeness of each sample compared to the SuperCOSMOS catalogue is given in parentheses (all galaxies brighter than the given limit) and brackets (galaxies brighter than the given limit, but fainter than the limit in the previous row).

\begin{tabular}{lrrl}
\hline \hline Field & $b_{j}$ Mag lim. & SuperCOSMOS & Velocities \\
\hline Full & $<17.0$ & 2981 & $2332(78 \%)$ \\
Full & $<17.5$ & 6331 & $3601(57 \%)[38 \%]$ \\
Full & $<18.0$ & 13361 & $4843(36 \%)[18 \%]$ \\
Full & $<18.5$ & 27177 & $5701(21 \%)[6 \%]$ \\
\hline Inter-cluster & $<17.0$ & 2404 & $1547(64 \%)$ \\
Inter-cluster & $<17.5$ & 5105 & $2411(47 \%)[32 \%]$ \\
Inter-cluster & $<18.0$ & 10862 & $2890(27 \%)[8 \%]$ \\
Inter-cluster & $<18.5$ & 21917 & $3233(15 \%)[3 \%]$ \\
\hline
\end{tabular}

$b_{j}<17.0$. Figure 1 shows the completeness of the Shapley velocity catalogue. Each of the panels corresponds to a different magnitude limit: 17, 17.5, 18, and 18.5. We used the HEALPix package $^{3}$ (Górski et al. 2005) to calculate the completeness mask. For each pixel of $2.6 \times 10^{-4}$ strd, we count galaxies up to a magnitude limit, defining the completeness in a pixel as the ratio of the number of galaxies with measured velocities to the number of SuperCOSMOS galaxies in the same pixel. The scale represents the fraction of galaxies in the SuperCOSMOS catalogue that have measured redshifts in the present velocity catalogue.

\subsection{Galaxy overdensity}

The present velocity catalogue allows us to calculate the overdensity in redshift space over our full survey region of $225 \mathrm{deg}^{2}$ and our intercluster region of $192 \mathrm{deg}^{2}$. As we are mostly interested in galaxies at the distance of the SSC, the overdensity is best seen as the peaks in the velocity histogram. We also computed the expected distribution for a smooth, homogeneous galaxy distribution based on the number count data of Metcalfe et al. (1991), allowing for the differential incompleteness of each sample as a function of $b_{j}$, as listed in the final column of Table 3 .

We calculated the galaxy overdensity as the ratio of the number of observed galaxies within the nominal velocity limits of the SSC complex (9000-18000 $\mathrm{km} \mathrm{s}^{-1}$ ) to the number expected from the Metcalfe counts within the same velocity limits, all with $b_{j}<17.5$, since, at the distance of the SSC, this magnitude limit contains essentially all galaxies that make a substantial contribution to the total luminosity. In the full region, the overdensity is ${ }^{4} 5.4 \pm 0.2$, and in the restricted intercluster region it is $3.8 \pm 0.2$. The surface overdensity in the area covered by this study (disregarding redshift information) is $2.9 \pm 0.2$.

\footnotetext{
http://www . eso.org/science/healpix/content/ HEALPix_reference.html

4 We give formal, statistical errors, not including any systematic effects. In particular, these overdensities are likely to be overestimates, as the galaxies have generally been selected in such a way as to maximize their chance of belonging to the SSC.
} 

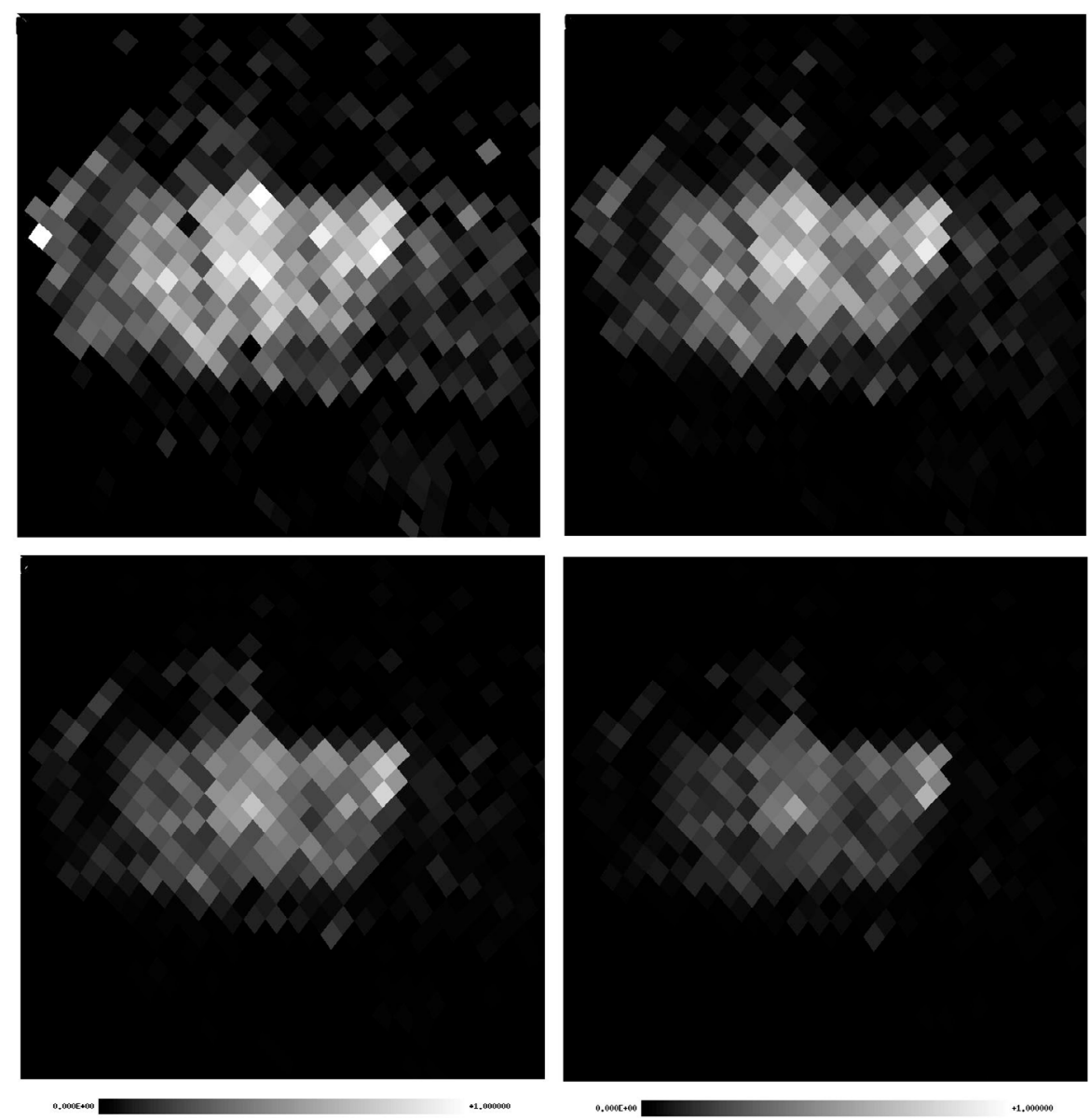

Fig. 1. Completeness of the Shapley velocity catalogue with different magnitude limits: $b_{j}=17.0$ (top left), 17.5 (top right), 18.0 (bottom left), and 18.5 (bottom right).

This strengthens earlier evidence for a very significant galaxy overdensity in the intercluster space of the SSC region (e.g., Bardelli et al. 2000). In terms of galaxy numbers, the intercluster galaxies make up $48 \%$ of the 3705 galaxies in the SSC velocity range. So, assuming a similar mass function and taking account of the lower completeness in the intercluster regions, these contribute nearly twice as much mass as the cluster galaxies.

In their study of the central part of the SSC, Bardelli et al. (2000) report an overdensity of $3.9 \pm 0.4$ for their intercluster sample and $11.3 \pm 0.4$ for their total sample, on scales of $10 h^{-1} \mathrm{Mpc}$. The latter value of the overdensity is much higher than ours, paper due to the much smaller scale. Drinkwater et al. (2004) found overdensities respectively $5.0 \pm 0.1$ and $3.3 \pm 0.1$ for a magnitude limit $R<17.0$ in a slightly less extended region of the SSC, of 26-40 $h^{-1} \mathrm{Mpc}$. These two sets of values are comparable, since $b_{j}-R \simeq 0.7$ in the direction of the SSC. They are higher than the mean density of luminous matter in the superclusters of the SDSS (Einasto et al. 2003a). The peak densities are in the range of 2.2-4.5, and the mean densities in the range of 1.9-2.8 as computed by Einasto (2005). Our data probe the distribution on much larger scales of around 12-18 degrees, corresponding to $30-46 h^{-1} \mathrm{Mpc}$, and not just in the denser, inner region. For the intercluster regions of the Horologium-Reticulum supercluster (HRS) on a large scale $40 h^{-1} \mathrm{Mpc}$, Fleenor et al. (2005) find an overdensity of 2.4. Their data and those from the present paper support the conclusion of Einasto et al. (2001) that the SSC and HRS constitute the two largest mass concentrations in the local universe.

\section{Velocity distribution and topology of the Shapley Supercluster}

The definition of the topology of the Shapley Supercluster is not an easy task, because of the complexity of the structures in the velocity distribution. The presence of many clusters, with their characteristic finger-of-God velocity structures, makes the study difficult. Moreover, remaining irregularities and gaps in the observations could mimic apparent structures. Finally, as modern redshift surveys show, dense structures are linked to each other by filaments and walls, forming a fabric that weaves throughout space.

\subsection{Velocity distribution}

Figure 2 shows the velocity distribution of galaxies in the direction of the Shapley Supercluster with all available 


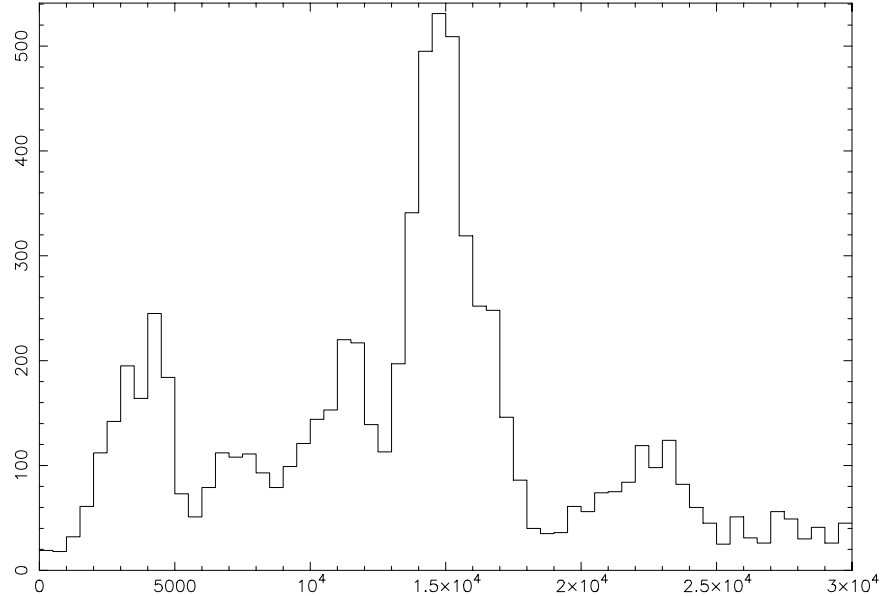

Fig. 2. Histogram of galaxy velocities in the direction of the Shapley Supercluster with all velocities available in the range $0 \mathrm{~km} \mathrm{~s}^{-1} \leq v \leq$ $30000 \mathrm{~km} \mathrm{~s}^{-1}$, with a step size of $500 \mathrm{~km} \mathrm{~s}^{-1}$.

velocities in the range $0 \leq v \leq 30000 \mathrm{~km} \mathrm{~s}^{-1}$, with a step size of $500 \mathrm{~km} \mathrm{~s}^{-1}$. The histogram presents four maxima, as discussed by Quintana et al. (2000). The main body of the SSC is represented by the highest peak, which is centered at $\simeq 15000 \mathrm{~km} \mathrm{~s}^{-1}$ and extends over the velocity range $13000-18000 \mathrm{~km} \mathrm{~s}^{-1}$. Another peak between 9000 and $13000 \mathrm{~km} \mathrm{~s}^{-1}$ shows the nearer concentration which is located to the East, centered on A3571. The other two peaks are at $4000 \mathrm{~km} \mathrm{~s}^{-1}$ (the Hydra-Centaurus region), and at $23000 \mathrm{~km} \mathrm{~s}^{-1}$, another possible supercluster behind the SSC. At higher redshifts, a few clumps of galaxies are also present.

Figure 3 shows the combined resulting distribution of galaxies towards the Shapley Supercluster as wedge diagrams in right ascension (top) and declination (bottom) for the whole velocity catalogue. The importance of the SSC in this region of the sky is demonstrated by the fact that $4212(50 \%)$ of the galaxies belong to the SSC and its inmediate neighborhood, if we consider as such all galaxies with velocities in the range 9000-18000 $\mathrm{km} \mathrm{s}^{-1}$ (a total depth of $90 \mathrm{~h}^{-1} \mathrm{Mpc}$ ). It can be seen that by probing large regions of the SSC away from the richer Abell clusters, we have confirmed significant additional structures which make complex links with the main cluster locations. The transverse dimensions of the area surveyed are defined from $\mathrm{RA} \simeq 12^{\mathrm{h}} 40^{\mathrm{m}}$ to $\simeq 14^{\mathrm{h}} 10^{\mathrm{m}}$ and from Dec $\simeq-24^{\circ}$ to $\simeq-38^{\circ}$, corresponding to at least $40 \times 25 h^{-1} \mathrm{Mpc}$.

\subsection{Structure of the Shapley Supercluster}

The larger velocity catalogue used in this analysis confirms the general structure and main features of the SSC already discussed in Quintana et al. (2000). For completeness, we summarize them here, pointing out new features that have surfaced. The central region (CR) is roughly spherical in shape and has at its core the highest-density, elongated volume containing the Abell clusters A3562, A3558 and A3556, with almost identical recession velocities around $14400 \mathrm{~km} \mathrm{~s}^{-1}$, and the groups SC1329-314 and SC1327-312, whose more discrepant velocities (by several hundred kilometers per second) could be attributed to the infall component along the line of sight. Towards the south of the elongated feature, the central region contains also the cluster A3560. As described in Reisenegger et al. (2000), the whole of this central region and all of its immediate surroundings are within the volume that is currently undergoing gravitational collapse.

We note the presence of a prominent foreground wall of galaxies (Hydra-Centaurus region) at $\bar{v}=4000 \mathrm{~km} \mathrm{~s}^{-1}$. This distribution can be related to the nearby cluster A3627, associated with the "Great Attractor" (Kraan-Korteweg et al. 1996). Moreover, a remarkable bridge of galaxies, groups and clusters, the so-called "Front Eastern Wall" (Quintana et al. 2000), extends to the east and in front of the supercluster, the densest part being at $\simeq 10000-11000 \mathrm{~km} \mathrm{~s}^{-1}$, located to the east. It contains the clusters A3571, A3572, A3575 and the group SC1336-314. The A3570 cluster is located at the southern tip of the observed part of the wall and A3578 at its northern one. This wall establishes a link between the Hydra-Centaurus region and the SSC, while a second one extends towards the west at $\bar{v}=8300 \mathrm{~km} \mathrm{~s}^{-1}$. Clumps of objects clearly link the two main structures. However, care must be taken in the interpretation of the wedge plots because of the finger-of-God effect evident in the main SSC concentrations (made especially prominent by the higher completeness in these cluster regions) and because of an analogous effect (with opposite sign) due to the inflow on larger scales, which makes the overdensities appear more overdense in redshift space than they are in the real space. Furthermore, the opening angle of the wedge diagram on the lower panel of Fig. 3 is enhanced by a factor of two, so the structures are stretched across the line of sight.

As is well known, a large concentration of galaxies and clusters at about RA $=12^{\mathrm{h}} 50^{\mathrm{m}}$ and around $v=$ 16000-17 $000 \mathrm{~km} \mathrm{~s}^{-1}$, the "A3528 complex" (Quintana et al. 1995; Bardelli et al. 2000) is connected to the main body of the SSC by a broad bridge of galaxies. It can also be seen from the wedge diagram in declination (Fig. 3, lower panel) that the Southern part of the SSC consists of two large sheets of galaxies where the known Abell clusters represent the peaks of maximum density. In this diagram, the more distant sheet, in particular, at $\bar{v}=15000 \mathrm{~km} \mathrm{~s}^{-1}$ is present right across the observed region from $-26^{\circ}$ up to $-38^{\circ}$, so the true extent of this wall is still currently unknown. The Southern part of this wall may be an extension of the plane of galaxies defined by Bardelli et al. (2000), since it has the same offset of $-5 h^{-1} \mathrm{Mpc}$ when analysed by Bardelli et al. in their Fig. 4.

\subsection{Connections of the SSC to surrounding superclusters}

Figure 3 also shows the presence of a large background complex of galaxies at $\bar{v}=22500 \mathrm{~km} \mathrm{~s}^{-1}$, as well as more distant clusters. This complex is associated with the supercluster SCL134 in the catalogue of Einasto et al. (2001). Background structures appear to be linked to the SSC by an extremely long and thin filament starting at RA $=13^{\mathrm{h}}$. In Fig. 4, this filament appears to extend out to $v=48000 \mathrm{~km} \mathrm{~s}^{-1}$, although its reality cannot be fully ascertained, given the non-uniform selection of 


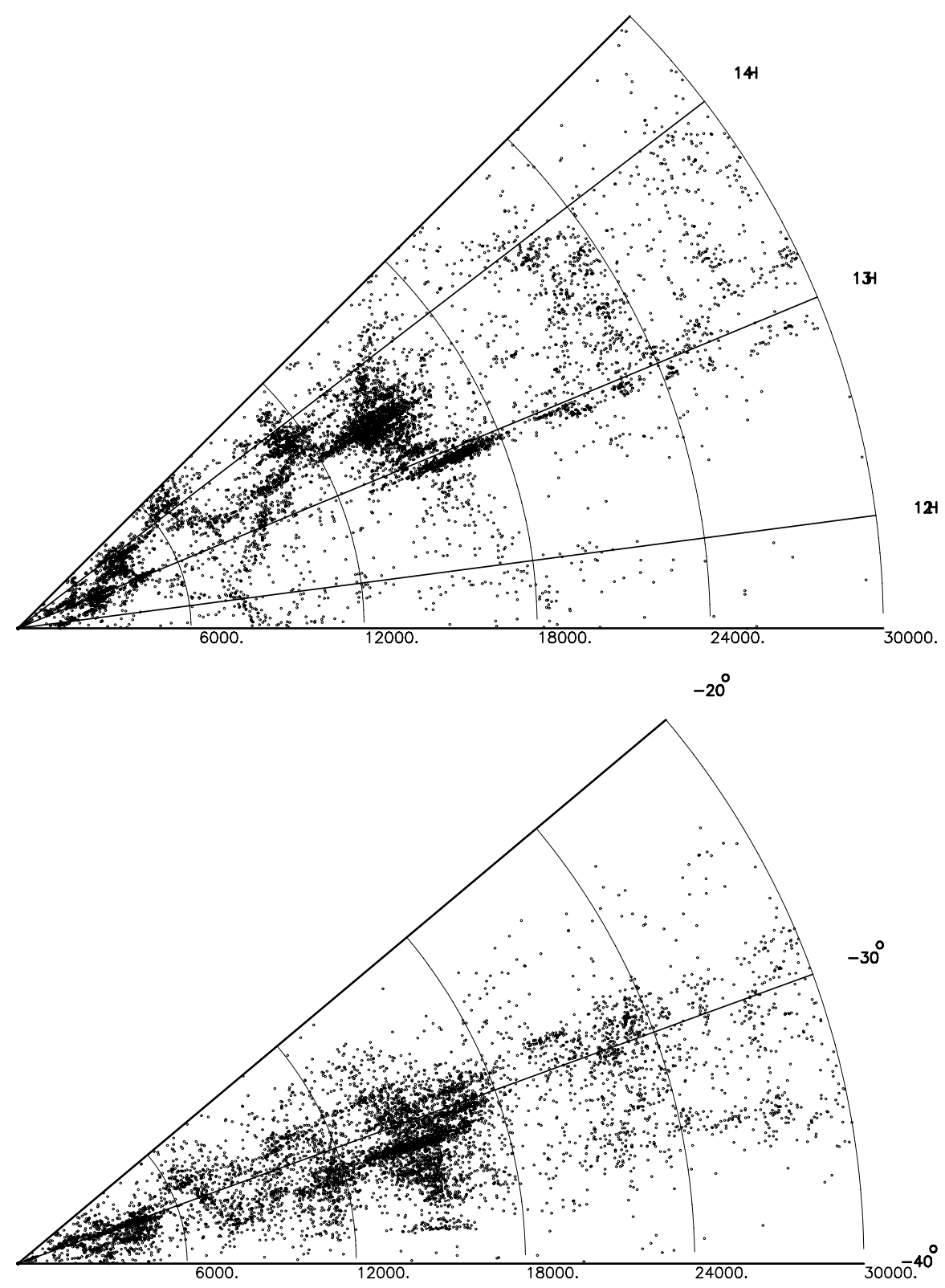

Fig. 3. Two projections of the distribution of galaxies with measured redshifts in the region of the Shapley Supercluster. The radial coordinate, the recession velocity determined from the redshift, $c z$, measured in $\mathrm{km} \mathrm{s}^{-1}$, is an imperfect surrogate (see text) for the galaxy's distance to us, who would be at the vertex. The angle in declination is expanded by a factor 2 relative to its true size for clarity.

galaxies and possible projection effects. However, two parts of this filament correspond to Einasto's superclusters SCL127 at $\bar{v}=22700 \mathrm{~km} \mathrm{~s}^{-1}$ and SCL129 at $\bar{v}=28300 \mathrm{~km} \mathrm{~s}^{-1}$. The two superclusters SCL146 and SCL266 also seem to be associated with radial extensions of the SSC.

The Shapley Supercluster is clearly linked to other huge superstructures, as shown in the Figs. 9 and 10 of Jones et al. (2004). Apart from the radial connection to the HydraCentaurus complex, a tangent bridge of galaxies extends in the direction of the Sextans supercluster at $z \simeq 0.04$. This distribution of galaxies across the southern sky, projected across the full range of southern declinations and each of the individual $10^{\circ}$ ranges in Jones et al. (2004), shows the very large number of ramifications connecting already identified structures between $\mathrm{RA}=10^{\mathrm{h}}$ and $15^{\mathrm{h}}$.

\section{Clusters of galaxies in the direction of the Shapley Supercluster}

Velocity dispersions are an essential piece of information to study cluster dynamics, because they directly probe the cluster potential. For a preliminary study (to be refined in Quintana et al. 2005), we searched the NED/IPAC Extragalactic Database for all clusters in the area defined by $12^{\mathrm{h}} 30^{\mathrm{m}}<\mathrm{RA}<$ $14^{\mathrm{h}} 30^{\mathrm{m}}$ and $-20^{\circ}>$ Dec $>-40^{\circ}$ (30 degrees by 20 degrees on the sky). For clusters with known velocities, we limited our search to $c z<40000 \mathrm{~km} \mathrm{~s}^{-1}$. The output list contains 141 clusters, 134 of which are listed in the ACO catalog, four are previously identified groups of galaxies (Quintana et al. 2000), two are X-ray detected clusters (RXC J1304.2-3030 by 


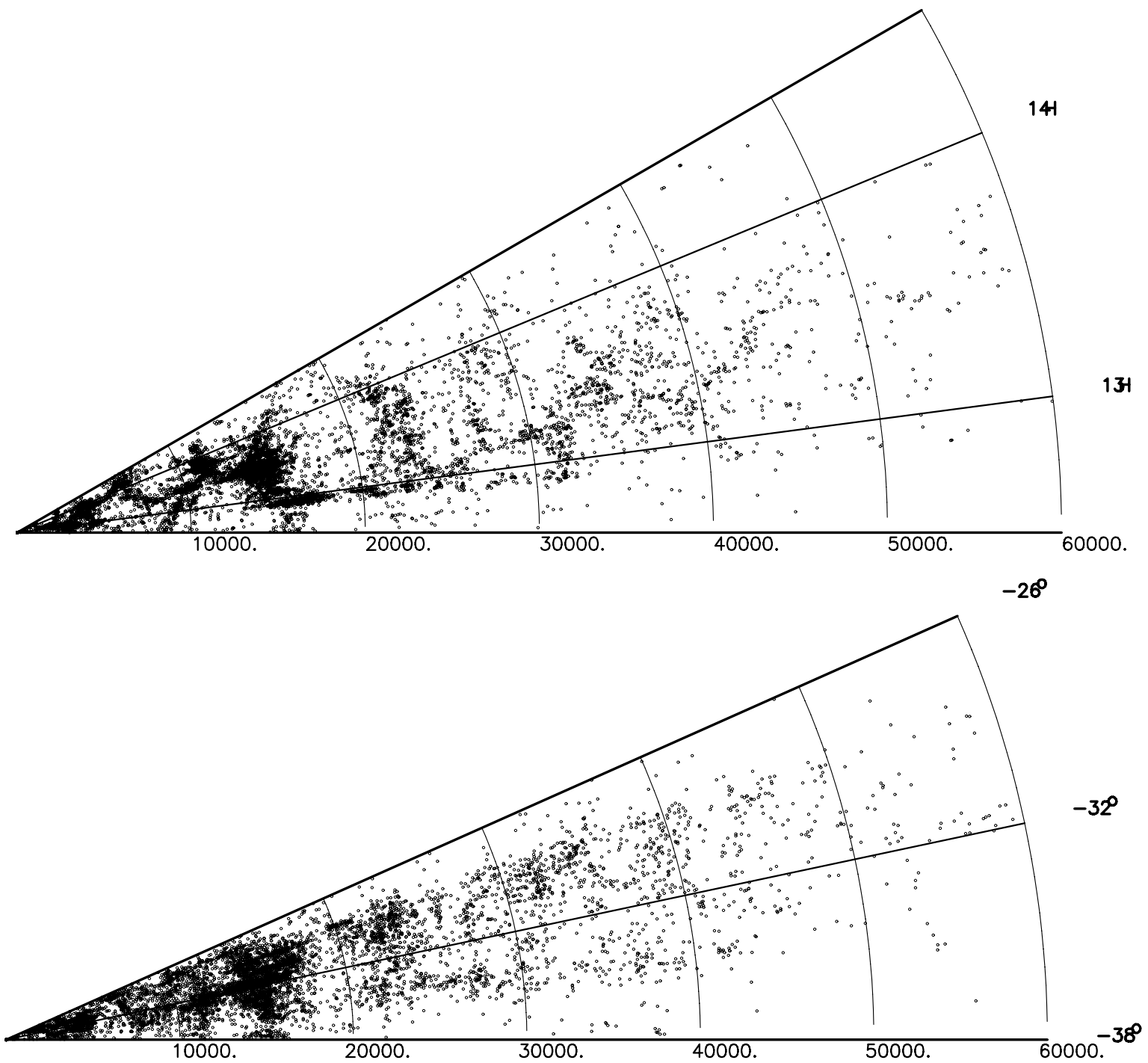

Fig. 4. Wedge diagrams in right ascension and declination extending out to $v=60000 \mathrm{~km} \mathrm{~s}^{-1}$, which suggest the presence of a long, thin filament starting at the SSC at $\mathrm{RA}=13^{\mathrm{h}}$ and extending out to $v=48000 \mathrm{~km} \mathrm{~s}^{-1}$.

Böhringer et al. 2004, RX J1252.5-3116 by Pierre et al. 1994), and one, CL 1322-30, is a cluster detected by Stein (1996).

The cluster centers were chosen by one of the following criteria: (a) from published X-ray centers; (b) from the position of the brightest cluster galaxy (cD or D if present); (c) using the Abell cluster center. For clusters with known velocities, most of the centers listed in the NED correspond to the bulk of the Shapley Supercluster and are given by Quintana et al. (2000). For all clusters with unknown velocities, we used the Abell cluster center.

As a relatively rough, first approach, we examined the velocity distribution of galaxies within a common angular radius of 0.5 degree of each cluster center (corresponding to $1.3 h^{-1} \mathrm{Mpc}$, roughly an Abell radius, at $z=0.05$, but smaller or larger for foreground or background clusters, respectively). The average recession velocity, $\langle V\rangle$, and the one-dimensional line-of-sight velocity dispersion, $\sigma_{V}$, were calculated using the bi-weighted estimators of location and scale of Beers et al. (1990). We used an iterative procedure by calculating the location and scale using the ROSTAT program and applying a
$3 \sigma$-clipping algorithm to the results. We repeated this procedure until the velocity dispersion converged to a constant value (within two or three iterations).

The number of clusters with at least six galaxies in our velocity sample is 68 . Forty-four clusters are in the velocity range of the Shapley Supercluster $\left(9000 \mathrm{~km} \mathrm{~s}^{-1}<c z<\right.$ $18000 \mathrm{~km} \mathrm{~s}^{-1}$ ) and eight are foreground. In the velocity interval of $18000 \mathrm{~km} \mathrm{~s}^{-1}<c z<30000$, we identified 11 clusters. The values of $\langle V\rangle$ and $\sigma_{V}$ for individual clusters are presented in Table $4 . N_{\text {sel }}$ is the number of galaxies selected within $0.5 \mathrm{de}$ gree and $N_{\text {mem }}$ is the number of member galaxies. The identification of the cluster as a member of a particular supercluster is indicated in the comments column with the designation of Einasto et al. (2001). SCL124 and SCL128 correspond, respectively, to the SSC and the Hydra-Centaurus supercluster.

Many clusters present significant substructures, identified by the multi-modality of their velocity distributions. In some cases, the main structures associated with catalogued clusters are behind (e.g. A3524, A3531, A3535, AS717, A3546, AS725, A3549, A3551, A3557, SC1340-294, A3576) or in 
Table 4. Clusters of galaxies in the area of the Shapley Supercluster.

\begin{tabular}{|c|c|c|c|c|c|c|c|}
\hline Cluster & $\begin{array}{c}\text { RA (J2000) } \\
\text { h m s } \\
(2)\end{array}$ & $\begin{array}{c}\operatorname{Dec}(2000) \\
\circ, " 1 \\
(3)\end{array}$ & $\begin{array}{l}N_{\text {sel }} \\
\text { (4) }\end{array}$ & $\begin{array}{r}N_{\text {mem }} \\
(5)\end{array}$ & $\begin{array}{l}\langle V\rangle \\
\mathrm{km} \mathrm{s}^{-1} \\
(6)\end{array}$ & $\begin{array}{l}\sigma_{V} \\
\mathrm{~km} \mathrm{~s}^{-1} \\
(7)\end{array}$ & $\begin{array}{l}\text { Comments } \\
\text { (8) }\end{array}$ \\
\hline A3524 & 124005.3 & -341328 & 19 & 9 & $22009 \pm 171$ & $465 \pm 165$ & background \\
\hline RX J1252.5-3116 & 125230.0 & -311600 & 11 & 6 & $16110 \pm 206$ & $425 \pm 102$ & X-ray center (Pierre at al. 1994) \\
\hline A3528 & 125418.2 & -290121 & 198 & 53 & $16294 \pm 100$ & $729 \pm 109$ & SCL124 \\
\hline A3530 & 125536.9 & -302120 & 66 & 35 & $16253 \pm 100$ & $583 \pm 65$ & $r_{\text {sel }}<13^{\prime}$ SCL124 \\
\hline A3531 & 125708.2 & -325519 & 33 & 17 & $22779 \pm 102$ & $404 \pm 115$ & SCL127 \\
\hline A3532 & 125719.2 & -302218 & 67 & 60 & $16667 \pm 87$ & $670 \pm 86$ & $r_{\text {sel }}<13^{\prime}$ SCL124 \\
\hline A3535 & 125748.6 & -282917 & 105 & 56 & $20213 \pm 58$ & $436 \pm 26$ & background \\
\hline AS717 & 125804.7 & -281111 & 97 & 10 & $22668 \pm 124$ & $362 \pm 89$ & background \\
\hline AS718 & 125945.0 & -334015 & 73 & 14 & $14527 \pm 68$ & $243 \pm 50$ & \\
\hline A3537 & 130102.7 & -322614 & 44 & 12 & $9371 \pm 76$ & $248 \pm 133$ & SCL128 \\
\hline RXC J1304.2-3030 & 130416.7 & -303055 & 52 & 29 & $3318 \pm 64$ & $331 \pm 36$ & $\begin{array}{l}\text { X-ray (Böhringer et al. 2004) } \\
\text { foreground }\end{array}$ \\
\hline AS721 & 130606.0 & -373511 & 43 & 36 & $14876 \pm 116$ & $686 \pm 72$ & \\
\hline A 3542 & 130841.0 & -343359 & 46 & 6 & $10528 \pm 49$ & $373 \pm 84$ & SCL124 \\
\hline A3542a & & & & 7 & $15146 \pm 100$ & $233 \pm 44$ & \\
\hline A $3542 b$ & & & & 13 & $27087 \pm 252$ & $859 \pm 150$ & background \\
\hline A3544 & 131104.7 & -325956 & 26 & 11 & $14969 \pm 164$ & $502 \pm 140$ & \\
\hline A3545 & 131123.3 & -340457 & 49 & 13 & $29879 \pm 142$ & $484 \pm 112$ & SCL129C \\
\hline A3545a & & & & 8 & $27190 \pm 165$ & $411 \pm 137$ & \\
\hline A3546 & 131303.7 & -295855 & 44 & 14 & $32113 \pm 77$ & $275 \pm 40$ & SCL129C \\
\hline AS724 & 131317.0 & -325655 & 39 & 12 & $14864 \pm 157$ & $510 \pm 85$ & \\
\hline AS724a & & & & 17 & $30401 \pm 150$ & $597 \pm 105$ & background \\
\hline AS725 & 131409.8 & -301153 & 18 & 9 & $32172 \pm 92$ & $250 \pm 80$ & background \\
\hline A3549 & 131421.5 & -292653 & 36 & 8 & $22878 \pm 173$ & $432 \pm 133$ & SCL127 \\
\hline A3549b & & & & 11 & $31300 \pm 174$ & $953 \pm 114$ & \\
\hline AS726 & 131511.7 & -333852 & 34 & 19 & $14892 \pm 137$ & $578 \pm 77$ & \\
\hline A3551 & 131810.8 & -305546 & 21 & 12 & $37434 \pm 294$ & $952 \pm 243$ & background \\
\hline A3552 & 131853.5 & -314848 & 47 & 34 & $14753 \pm 119$ & $682 \pm 60$ & SCL124 \\
\hline A3553 & 131914.6 & -371045 & 20 & 12 & $15141 \pm 153$ & $497 \pm 95$ & SCL124 \\
\hline A3554 & 131930.5 & -332845 & 61 & 36 & $14431 \pm 94$ & $560 \pm 66$ & SCL124 \\
\hline A3555 & 132046.2 & -285847 & 45 & 22 & $14034 \pm 47$ & $214 \pm 33$ & SCL124 \\
\hline AS729 & 132132.2 & -354744 & 55 & 20 & $15158 \pm 106$ & $462 \pm 102$ & \\
\hline
\end{tabular}

front of (e.g. RXC J1304.2-3030, CL1322-30, A3565, A3574, AS753, A3581, AS761) the Shapley supercluster.

Most of the clusters are well isolated in their threedimensional distribution, with some exceptions. Four clusters in the sample present significant foreground and background structures (A3542, A3545, AS724, AS731). For these clusters, $\langle V\rangle$ and $\sigma_{V}$ are reliable values, however a more detailed analysis is required to determine: (a) where the cluster is located; (b) if the foreground and background structures seen in the $3 \mathrm{D}$ distribution are real.

The distribution on the sky of the clusters and galaxies in the velocity range of the Shapley Supercluster is presented in Fig. 5. At $\simeq 9000-13000 \mathrm{~km} \mathrm{~s}^{-1}$, two main cluster clumps are present (left panel). The first one is formed by the clusters A3571, A3572, A3575m AS748 and AS744 (the "Front Eastern Wall", see Sect. 4.2) and the second is formed by the clusters A1736a and AS736. At $\simeq 13000-18000 \mathrm{~km} \mathrm{~s}^{-1}$, it is possible to see three main cluster clumps: the core of the SSC, formed by the clusters A3552, A3556, A3558, and A3562 and the groups SC1329-314 and SC1327-312, and the two extensions formed by A3568, A3566, and A3464 and by A3528, A3530, and A3532, respectively (see Sect. 4.2).

\section{Global properties}

Ignoring redshift-space distortions due to peculiar velocities, the surveyed area of $225 \mathrm{deg}^{2}$, from 9000 to $18000 \mathrm{~km} \mathrm{~s}^{-1}$, corresponds to a spatial volume $V_{\mathrm{SSC}}=1.17 \times 10^{5} \mathrm{~h}^{-3} \mathrm{Mpc}^{3}$, equivalent to that of a sphere of radius $r_{\mathrm{eff}}=30.3 \mathrm{~h}^{-1} \mathrm{Mpc}$. This is near the middle of the range of supercluster sizes found by Einasto et al. (2003a,b) in their systematic searches of the Sloan Digital Sky Survey (SDSS) and Las Campanas Redshift Survey (LCRS) data. However, we note that their density threshold for selecting superclusters is 1.8 times the average redshift-space density, much lower than the overdensity of 5.4 we find in our study. This means that the true extension of the Shapley Supercluster, applying the criterion of Einasto and collaborators, would be much larger than the area of our study. In the extreme case of having an empty volume around the region of our study, the radius enclosing an average overdensity of 1.8 is $(5.4 / 1.8)^{1 / 3} r_{\text {eff }} \simeq 44 h^{-1} \mathrm{Mpc}$, increasing to $\simeq 54 h^{-1} \mathrm{Mpc}$ if the surrounding volume is assumed to be of average density, which is probably still an underestimate. 
Table 4. continued.

\begin{tabular}{|c|c|c|c|c|c|c|c|}
\hline Cluster & $\begin{array}{c}\text { RA (J2000) } \\
\mathrm{h} \mathrm{m} \mathrm{s} \\
(2)\end{array}$ & $\begin{array}{c}\overline{D e c}(2000) \\
0^{\prime}, " \\
(3)\end{array}$ & $\overline{N_{\text {sel }}}$ & 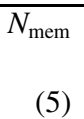 & $\begin{array}{l}\langle V\rangle \\
\mathrm{km} \mathrm{s}^{-1} \\
(6)\end{array}$ & $\begin{array}{l}\sigma_{V} \\
\mathrm{~km} \mathrm{~s}^{-1} \\
(7)\end{array}$ & $\begin{array}{l}\text { Comments } \\
\text { (8) }\end{array}$ \\
\hline AS731 & 132301.9 & -345241 & 104 & 38 & $15230 \pm 84$ & $514 \pm 61$ & \\
\hline AS731a & & & & 17 & $7940 \pm 85$ & $339 \pm 69$ & \\
\hline A3556 & 132406.2 & -313944 & 123 & 75 & $14439 \pm 71$ & $618 \pm 50$ & SCL124 \\
\hline CL1322-30 & 132447.6 & -301738 & 56 & 21 & $4242 \pm 64$ & $287 \pm 46$ & $\begin{array}{l}\text { detected by Stein (1996) } \\
\text { foreground }\end{array}$ \\
\hline A 3557 & 132452.8 & -285243 & 59 & 22 & $23401 \pm 75$ & $343 \pm 73$ & SCL 127 \\
\hline AS733 & 132629.3 & -365807 & 22 & 8 & $14908 \pm 167$ & $417 \pm 77$ & \\
\hline A1736a & 132644.3 & -272622 & 264 & 92 & $10215 \pm 47$ & $450 \pm 30$ & \\
\hline A1736b & 132648.7 & -270838 & 264 & 143 & $13654 \pm 73$ & $873 \pm 52$ & \\
\hline A 3558 & 132756.9 & -312944 & 285 & 247 & $14307 \pm 64$ & $1010 \pm 44$ & SCL124 \\
\hline SC1327-312 & 132947.0 & -313629 & 48 & 35 & $14650 \pm 147$ & $857 \pm 117$ & $\begin{array}{l}\text { X-ray center (Breen et al. 1994) } \\
r_{\text {sel }}<11^{\prime}\end{array}$ \\
\hline A3559 & 132951.0 & -293051 & 168 & 82 & $14130 \pm 57$ & $519 \pm 45$ & SCL124 \\
\hline AS736 & 133059.2 & -280226 & 64 & 30 & $10140 \pm 95$ & $514 \pm 70$ & \\
\hline SC1329-314 & 133136.0 & -314846 & 38 & 35 & $13541 \pm 151$ & $883 \pm 168$ & $\begin{array}{l}\text { X-ray center (Breen et al. 1994) } \\
r_{\text {sel }}<9^{\prime}\end{array}$ \\
\hline A 3560 & 133225.3 & -330812 & 73 & 56 & $14551 \pm 106$ & $793 \pm 116$ & SCL124 \\
\hline A 3562 & 133356.8 & -312923 & 41 & 40 & $14455 \pm 191$ & $1197 \pm 194$ & $r_{\mathrm{sel}}<12^{\prime} \mathrm{SCL} 124$ \\
\hline A3564 & 133422.4 & -351322 & 64 & 30 & $15116 \pm 73$ & $393 \pm 64$ & SCL124 \\
\hline SC1336-314 & 133619.0 & -314800 & 85 & 7 & $11982 \pm 189$ & $941 \pm 261$ & \\
\hline A 3565 & 133639.8 & -335818 & 27 & 13 & $3860 \pm 43$ & $148 \pm 118$ & SCL128 \\
\hline A3566 & 133859.4 & -353313 & 42 & 25 & $15388 \pm 106$ & $519 \pm 58$ & SCL124 \\
\hline SC1340-294 & 134000.0 & -294559 & 26 & 15 & $23226 \pm 126$ & $467 \pm 75$ & background \\
\hline A3568 & 134131.0 & -342400 & 39 & 27 & $15218 \pm 143$ & $726 \pm 101$ & \\
\hline SC1342-302 & 134226.4 & -301926 & 38 & 14 & $14621 \pm 105$ & $375 \pm 49$ & New cluster \\
\hline AS739 & 134253.7 & -345806 & 29 & 8 & $15318 \pm 243$ & $607 \pm 134$ & \\
\hline AS742 & 134436.0 & -341800 & 53 & 22 & $15124 \pm 100$ & $455 \pm 93$ & \\
\hline AS744 & 134728.4 & -320857 & 48 & 31 & $11882 \pm 138$ & $756 \pm 118$ & \\
\hline A3571 & 134728.9 & -325158 & 95 & 92 & $11676 \pm 106$ & $1016 \pm 80$ & SCL124 \\
\hline A 3572 & 134809.7 & -332205 & 14 & 7 & $11740 \pm 279$ & $651 \pm 278$ & $r_{\text {sel }}<13^{\prime}$ SCL124 \\
\hline A 3574 & 134909.4 & -301755 & 57 & 38 & $4512 \pm 90$ & $549 \pm 56$ & SCL128 \\
\hline AS746 & 134949.0 & -345852 & 35 & 12 & $14877 \pm 123$ & $399 \pm 92$ & \\
\hline AS748 & 135235.5 & -322348 & 27 & 13 & $11866 \pm 97$ & $332 \pm 97$ & \\
\hline A 3575 & 135235.8 & -325247 & 39 & 20 & $11405 \pm 122$ & $531 \pm 92$ & SCL124 \\
\hline A 3576 & 135245.9 & -301747 & 51 & 21 & $22114 \pm 64$ & $283 \pm 42$ & background \\
\hline A 3577 & 135420.4 & -275045 & 37 & 24 & $14890 \pm 131$ & $628 \pm 98$ & SCL124 \\
\hline A 3578 & 135730.2 & -243305 & 16 & 9 & $11297 \pm 150$ & $407 \pm 79$ & SCL124 \\
\hline AS753 & 140338.5 & -335824 & 19 & 15 & $3960 \pm 84$ & $312 \pm 143$ & foreground \\
\hline A3581 & 140727.5 & -270116 & 32 & 24 & $6725 \pm 120$ & $574 \pm 56$ & SCL128 \\
\hline AS761 & 141847.2 & -272547 & 17 & 12 & $6902 \pm 153$ & $495 \pm 115$ & foreground \\
\hline
\end{tabular}

An estimate of the total luminosity, based on the assumption that the surveyed galaxies are distributed as the total light, can be given as

$L_{\mathrm{tot}}=($ overdensity $) \times \bar{j} \times V_{\mathrm{SSC}} \simeq 1.4 \times 10^{14} h^{-2} L_{\odot}$,

where the average $b_{j}$-band luminosity of the Universe, $\bar{j} \simeq$ $2.3 \times 10^{8} h L_{\odot} \mathrm{Mpc}^{-3}$, was obtained from the $2 \mathrm{dF}$ luminosity function of Norberg et al. (2002), and corresponds to an average mass-to-light ratio of $\simeq 360\left(\Omega_{\mathrm{M}} / 0.3\right) h\left(M_{\odot} / L_{\odot}\right)$. An alternative estimate, strictly speaking a lower bound, is the "observed luminosity", namely the sum of the luminosities of all the galaxies in our catalogue within the specified volume,

$L_{\text {obs }}=2.2 \times 10^{13} h^{-2} L_{\odot}$,

about 7 times smaller than our estimate for $L_{\text {tot }}$. This is not surprising, as our catalogue is not complete, and the total luminosity is strongly dominated by a few of the brightest galaxies, some of which may have been excluded from the surveys under the assumption that they were foreground objects, not belonging to the SSC.

These values, although still underestimates for the true luminosity of the SSC, because of the limited well-sampled volume, are higher than any of the corresponding luminosities of 

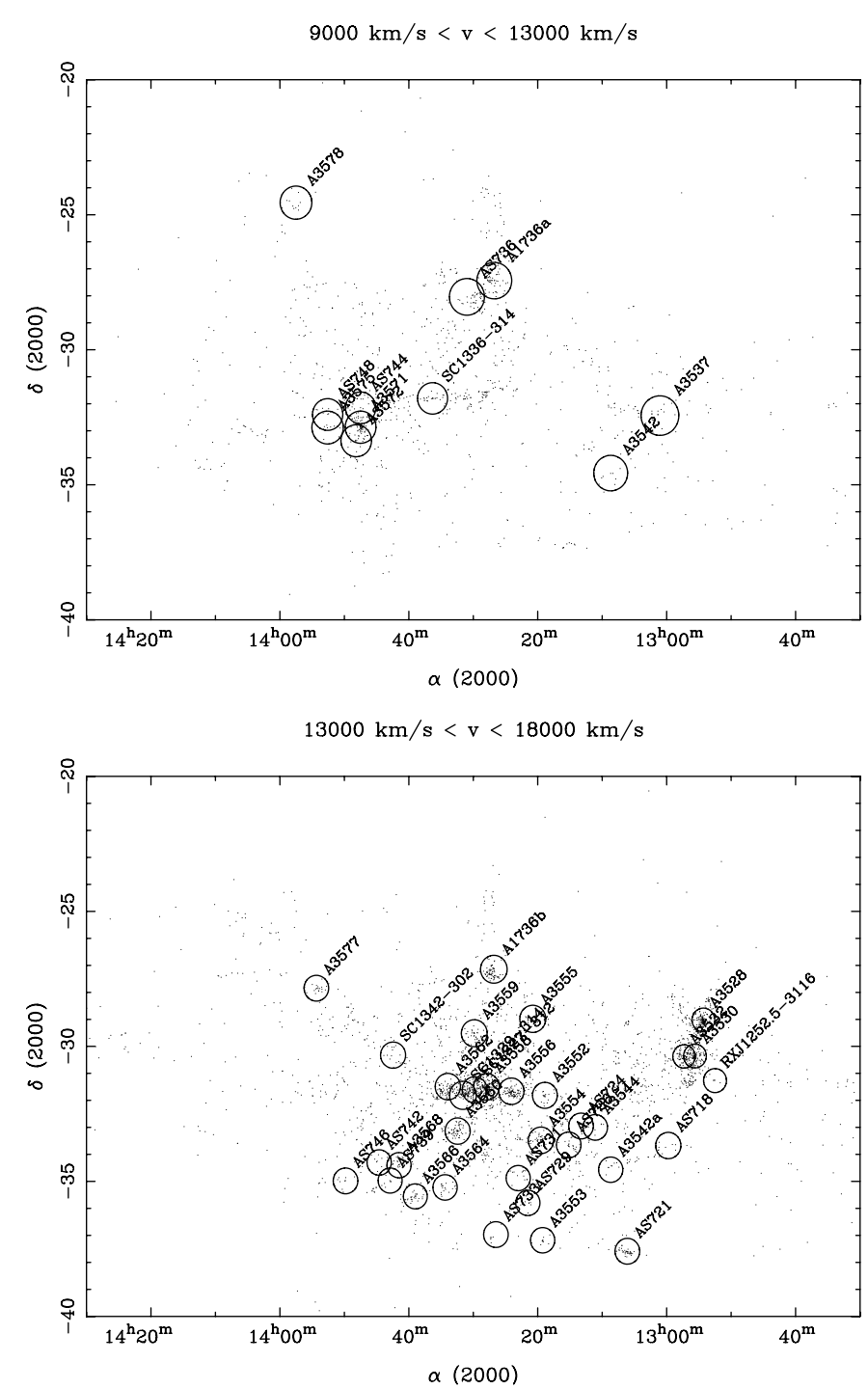

Fig. 5. Distribution on the sky of the 44 clusters and galaxies in the velocity range of the SSC. Upper panel: cluster with velocities between $9000 \mathrm{~km} \mathrm{~s}^{-1}$ and $13000 \mathrm{~km} \mathrm{~s}^{-1}$. Lower panel: clusters between $13000 \mathrm{~km} \mathrm{~s}^{-1}$ and $18000 \mathrm{~km} \mathrm{~s}^{-1}$.

the superclusters in the SDSS-South and comparable to the brightest in the SDSS-North and the LCRS, which are at substantially higher redshifts than the SSC (Einasto et al. 2003a,b). This confirms that the SSC is a truly exceptional structure, as found by previous surveys of the local Universe (e.g., Einasto et al. 2001).

Similarly, we can assume that the surveyed galaxies trace mass and estimate the total mass within the surveyed volume as

$M_{\text {tot }}=($ overdensity $) \times \Omega_{\mathrm{M}} \rho_{\text {crit }} V_{\mathrm{SSC}}=5 \times 10^{16} h^{-1} M_{\odot}$,

where $\rho_{\text {crit }}$ is the critical density for a flat Universe, and we assumed that the standard mass-density parameter is $\Omega_{\mathrm{M}}=0.3$. A lower bound on this mass, in essentially the same volume, was obtained by Ragone et al. (2005), who identified galaxy systems and estimated their individual masses. Adding these, they find $M_{\mathrm{obs}}=4.8 \times 10^{15} h^{-1} M_{\odot}$. Correcting (based on simulations) for systems presumably missed in the survey (mainly because of non-uniform incompleteness), this value increases to $M_{\text {corr }}=1.1 \times 10^{16} h^{-1} M_{\odot}$, about $1 / 4$ of our $M_{\text {tot }}$. If real and not due to biases of one or the other method, this would indicate that in fact a large fraction of the SSC $(80 \%$, if taken at face value) is not in galaxy clusters or groups.

Another dynamical estimate for the mass was given by Reisenegger et al. (2000), who searched for "caustics" in the projected radius-redshift diagram of the SSC and applied to these both a straightforward spherical collapse model (Regös \& Geller 1989) and the heuristic escape-velocity method of Diaferio (1999). The mass within a radius $r=8 h^{-1} \mathrm{Mpc}$ was found to be $\sim 10^{16} h^{-1} M_{\odot}$ in the former, and $\sim 2 \times 10^{15} h^{-1} M_{\odot}$ in the latter, corresponding to overdensities $\rho /\left(\Omega_{\mathrm{M}} \rho_{\text {crit }}\right) \simeq 60$ and 10 , respectively. A lower limit on the virialized mass in clusters turned out to be similar to the latter result. These results, based on a much smaller volume, are therefore hard to compare, but do not seem inconsistent with those of the present study.

The mass required at the distance of the SSC to produce the observed motion of the Local Group with respect to the CMB is $M_{\text {dipole }} \approx(1-3) \times 10^{17}\left(\Omega_{\mathrm{M}} / 0.3\right)^{0.4} h^{-1} M_{\odot}$ (Hoffman et al. 2001), not very far above the total mass of the SSC as estimated here. In consistent models (e.g. Branchini et al. 1999) of the density and velocity distribution on large scales (where density fluctuations are small) in the local Universe, the SSC figures prominently, although the Local Group motion originates from a combination of several attractors.

\section{Conclusions}

This paper is based on the largest velocity catalogue available up to now to analyse various properties of the Shapley Supercluster. It shows a completeness analysis for 8632 galaxies with measured velocities (10529 velocity measurements), including 1201 new velocities for 1166 galaxies to be published in Quintana et al. (2005). The completeness is highest for the brighter magnitude limits, peaking at $78 \%$ for the whole sample at $b_{j}<17.0$. The galaxy overdensity in the full SSC region is $\sim 5.4$, and in the restricted intercluster region it is $\sim 3.8$. These results place the SSC among the largest of superclusters found in recent, systematic surveys at higher redshifts. Its inferred mass is large enough to have a non-negligible effect on the observed Local Group motion. The SSC has a general flat morphology, extending further than all previous estimates and linking the foreground Hydra-Centaurus region. We also presented and discussed a preliminary catalogue of 68 galaxy clusters with dynamical information in this area of the sky, 44 of which are in the redshift range of the SSC.

Acknowledgements. This research was partially supported by the cooperative programme ECOS/CONICYT C96U04. DP and ES thank the Astronomy Department at PUC (Santiago de Chile) for its hospitality. DP acknowledges receipt of a France-Australie PICS in support of visits to Siding-Spring Observatory and the PICS-CNPq FranceBrazil cooperation $910068 / 00-3$ in support of visits to the University of Sao Paulo (IAG/USP). HQ was supported by the FONDAP Centre for Astrophysics. ERC is supported by the Gemini Observatory, which is operated by the Association of Universities for Research in Astronomy Inc., on behalf of the international Gemini partnership of Argentina, Australia, Brazil, Canada, Chile, the UK and the United 
States of America. A.R. and R.D. received support from FONDECYT through its Regular Project 1020840. We thank our referee, Prof. J. Einasto, for his precious remarks and suggestions. Some of the results in this paper have been derived using the HEALPix package (Górski et al. 2005)

\section{References}

Baldi, A., Bardelli, S., \& Zucca, E. 2001, MNRAS, 324, 509

Bardelli, S., Zucca, E., Vettolani, G., et al. 1994, MNRAS, 267, 665

Bardelli, S., Zucca, E., Malizia, A., et al. 1996, A\&A, 305, 435

Bardelli, S., Zucca, E., Zamorani, G., Vettolani, G., \& Scaramella, R. 1998, MNRAS, 296, 599

Bardelli, S., Zucca, E., Zamorani, G., Moscardini, L., \& Scaramella, R. 2000, MNRAS, 312, 540

Bardelli, S., Zucca, E., \& Baldi, A. 2001, MNRAS, 320, 387

Branchini, E., Teodoro, L., Frenk, C. S., Schmoldt, I., Efstathiou, G., et al. 1999, MNRAS, 308, 1

Böhringer, H., Schuecker, P., Guzzo, L., et al. 2004, A\&A, 425, 367

Breen, J., Raychaudhury, S., Forman, W., \& Jones, C. 1994, ApJ, 424, 59

Colless, M. M., et al. (2dFGRS team) 2001, MNRAS, 328, 1039

Diaferio, A. 1999, MNRAS, 309, 610

Drinkwater, M. J., Proust, D., Parker, Q. A., Quintana, H., \& Slezak, E. 1998, 14th IAP conference "Wide Field Surveys in Cosmology", Paris, 26-29 May 1998, ed. S. Colombi, Y. Mellier, \& B. Raban (Éditions Frontières), 392

Drinkwater, M. J., Proust, D., Parker, Q. A., Quintana, H., \& Slezak, E. 1999, PASA, 16, 113

Drinkwater, M. J., Parker, Q. A., Proust, D., Slezak, E., \& Quintana, H. 2004, PASA, 21, 89

Einasto, M., Einasto, J., Tago, E., Muller, V., \& Andernach, H. 2001, AJ, 122, 2222

Einasto, J., Hütsi, G., Einasto, M., et al. 2003a, A\&A, 405, 425

Einasto, J., Einasto, M., Hütsi, G., et al. 2003b, A\&A, 410, 425

Einasto, J. 2005, private communication

Felenbok, P., Guerin, J., Fernandez, A., et al. 1997, Experimental Astronomy, 7, 65

Fleenor, M. C., Rose, J. A., Christiansen, W. A., et al. 2005 [arXiv:astro-ph/0505361]

Górski, K. M., Hivon, E., Banday, A. J., et al. 2005, ApJ, 622, 759

Hambly, N. C., Mac Gillivaray, H. T., Read, M. A., et al. 2001a, MNRAS, 326, 1279

Hambly, N. C., Irwin, M. J., \& MacGillivray, H. T. 2001b, MNRAS, 326,1295
Hambly, N. C., Davenhall, A. C., Irwin, M. J., \& MacGillivray, H. T. 2001c, MNRAS, 326, 1315

Hoffman, Y., Eldar, A., Zaroubi, S., \& Dekel, A. 2001 [arXiv: astro-ph/0102190]

Jones, D. H., Saunders, W., Colless, M., et al. 2004, MNRAS, 355, 747

Kaldare, R., Colless, M., Raychaudhury, S., \& Peterson, B. A. 2003, MNRAS, 339, 652

Kraan-Korteweg, R. C., Woudt, P. A., Cayatte, V., et al. 1996, Nature, 379,519

Kurtz, M. J., \& Mink, D. J. 1998, PASP, 110, 943

Lund, G. 1986, Optopus. Multiple object spectroscopy at the Cassegrain focus of the $3.6 \mathrm{~m}$ telescope, in ESO Operating Manual, Garching: European Southern Observatory (ESO)

Metcalfe, N., Shanks, T., Fong, R., \& Jones, L. R. 1991, MNRAS, 249,498

Norberg, P., et al. 2002, MNRAS, 336, 907

Oguri, M., Takahashi, K., Ichiki, K., \& Ohno, H. 2004 [arXiv: astro-ph/0410145]

Parker, Q. A., \& Watson, F. G. 1995, in Wide Field Spectroscopy and the Distant Universe, 35th Herstmonceux Conference, ed. S. J. Maddox, \& A. Aragon-Salamanca (Singapore: World Scientific), 33

Pierre, M., Böhringer, H., Ebeling, H., et al. 1994, A\&A, 290, 725

Quintana, H., Ramírez, A., Melnick, J., Raychaudhury, S., \& Slezak, E. 1995, AJ, 110, 463

Quintana, H., Melnick, J., Proust, D., \& Infante, L. 1997, A\&AS, 125, 247

Quintana, H., Carrasco, E. R., \& Reisenegger, A. 2000, AJ, 120, 511

Quintana, H., Proust, D., Carrasco, E. R., \& Reisenegger, A. 2005, in preparation

Ragone, C. J., Merchán, M., Muriel, H., et al. 2005, A\&A, submitted Raychaudhury, S. 1989, Nature, 342, 251

Regös, E., \& Geller, M. 1989, AJ, 98, 755

Reisenegger, A., Quintana, H., Carrasco, E. R., \& Maze, J. 2000, AJ, 120,523

Scaramella, R., Baiesi-Pillastrini, G, Chincarini, G., Vettolani, G., \& Zamorani, G. 1989, Nature, 338, 562

Stein, P. 1996, A\&AS, 116, 203

Smoot, G. F., Bennett, C. L., Kogut, A., et al. 1992, ApJ, 396, L1

Tody, D. 1993, in Astronomical Data Analysis Software and Systems II, ed. R. J. Hanisch, R. J. V. Brissenden, \& J. Barnes, ASP Conf. Ser., 52, 173

Yoshida, N., Sheth, R., \& Diaferio, A. 2001, MNRAS, 328, 669 